\title{
A pilot study to evaluate the prevalence of malnutrition in patients with Clostridium difficile (C. diff) infection
}

\author{
S. S. Wong ${ }^{1}$, J. O'Driscoll ${ }^{2}$, M. Weldon ${ }^{3}$ and C. Y. Yau ${ }^{4}$ \\ Departments of Nutrition and Dietetics ${ }^{1}$, Microbiology ${ }^{2}$ and Gastroenterology ${ }^{3}$ and ${ }^{4}$ Medicine of Older People, \\ Stoke Mandeville Hospital, Aylesbury HP21 8AL, UK
}

\begin{abstract}
Malnutrition is frequently reported in hospitalised adults and it is associated with significant adverse consequences in relation to health outcomes and healthcare expenditures. In 2006 the Healthcare Commission reported on C. diff outbreaks at Stoke Mandeville Hospital ${ }^{(1)}$, increasing the awareness of $C$. diff-associated complications amongst UK hospitals. However, there is presently no data relating to the prevalence of malnutrition in patients with $C$. diff infection. The aims of the present study were: to establish the prevalence of malnutrition by Malnutrition Universal Screening Tool (MUST) screening ${ }^{(2,3)}$ in adults with $C$. diff infection; to carry out anthropometric measurement of BMI and albumin levels; functional assessment of handgrip (HG) strength ${ }^{(4)}$; nutrient intake using food record chart. During 2007-8 seventy-six patients aged between 18 and 103 years admitted to a district general hospital with hospital-acquired $C$. diff infection were assessed after having obtained local ethics approval. The present study found that the prevalence of undernutrition was $57 \%$ (MUST 2) at the time of $C$. diff diagnosis. In total $26 \%$ of patients had a BMI $<20 \mathrm{~kg} / \mathrm{m}^{2}, 87 \%$ of patients were found to have a hypoalbuminaemia (albumin $<30 \mathrm{~g} / \mathrm{l}$ ) and $73 \%$ of patients were found to have eaten less than half their meal at the time of diagnosis. Malnourished patients (MUST 2) were found to have a significantly lower HG strength (MUST 2, $45.5 \%$ reference value $v$. MUST $0,98.5 \%$ reference value; $P=0.0015$ ). No difference was found in relation to albumin levels (MUST 2, $22 \mathrm{~g} / \mathrm{l} v$. MUST 0, 22.5 g/l; $P=0.34$ ) when compared with well-nourished patients (MUST 0). The prevalence of malnutrition in patients with $C$. diff infection appears to be common in hospital and may be a risk factor for infection. Special attention should be given to vulnerable patients in hospital. A follow-up study with a larger sample size is underway to assess whether or not nutritional support improves patient clinical outcomes.

1. Health Care Commission (2006) Investigation into Outbreaks of Clostridium difficile at Stoke Mandeville Hospital, Buckinghamshire Hospitals NHS Trust. London: Health Care Commission.

2. Elia M (2003) Screening for Malnutrition: A Multidisciplinary Responsibility. Development and Use of the Malnutrition Universal Screening Tool (MUST) for Adults. Redditch, Worcs.: BAPEN.

3. National Institute for Health and Clinical Excellence (2006) Nutrition Support in Adults: Oral Nutrition Support, Enteral Tube Feeding and Parenteral Nutrition. London: NICE.

4. Klidjian AM, Forster KJ, Kammerling RM et al. (1980) Br Med J 281, 889-901.
\end{abstract}

